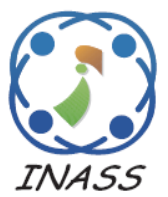

\title{
DOA Estimation Exploiting Moving Platform of Unfolded Coprime Array
}

\author{
Fatimah Abdulnabi Salman ${ }^{1 *}$ \\ Bayan Mahdi Sabbar ${ }^{1}$ \\ ${ }^{1}$ Department of System Engineering, Al-Nahrain university, Baghdad, Iraq \\ * Corresponding author's Email: Faty.a.salman@gmail
}

\begin{abstract}
Coprime array configurations have occupied a significant concentration due to its properties like simplicity and ability to detect a higher number of targets with respect to the number of elements in an antenna array. Nevertheless, the constructed array still has some empty positions (holes) that need to be filled to enhance the degrees of freedom (DOFs). Motion array can successfully address this issue, fill some holes and expand the number of the lags by shifting the distance of the elements in the coprime array half the wavelength, thus it can detect a great amount of sources compared to a fixed array. In this paper, a new synthetic array aperture named "Enhanced Unfolded Coprime Array" (EUFCA) formed on the moving platform concept is proposed to address the problem of the empty positions and provide a central consecutive lag by generating a hole-free virtual array. The proposed array configuration enhances unfolded coprime array (UFCA) by changing the position of some physical elements. Formal expression of the uniform degrees of freedom ( $\mathrm{uDOF}$ ) and the unique lags of the synthetic array are derived. Simulation results present the achievable large number of UDOF that enhance the DOA estimation process by detects more source signals compared with other array structure designs. For 18 elements in EUCFA, with different (M,N) pair such as $(5,9)$ and $(6,7)$, the number of achievable uDOFs for moved array is 215 and 213 respectively comparing to fixed array that generate 58 and $54 \mathrm{uDoFs}$ respectively. The ratio between the contiguous lags before and after movement is 3.71 and 3.94 for the aforementioned pairs respectively which indicate the ration of maximum number of extra contiguous lags obtained for EUFCA.
\end{abstract}

Keywords: Antenna array, Coprime array, Degree of freedom, Direction of arrival, Synthetic array.

\section{Introduction}

Direction of arrival (DOA) estimation is a vital research area in applications such as wireless communication, sonar, marina communications, and radar that produce a highly resolved spatial spectrum exploiting a uniform linear array (ULA) [1, 2]. Recently, sparse arrays have been exploited to perform DOA estimation since it can find more sources using a small number of physical elements. Sparse arrays, such as minimum redundant arrays (MRAs) [3] and minimum hole arrays (MHAs) [4] have been suggested to address the hole problem in the difference array, but they lack formal expression for the array design. Nested array [5] and coprime array [6] are the most popular arrays structure that make use of pairs of ULAs consists of $(N, M)$ pair of elements can generate up to $O(M N)$ uncorrelated sources using $M+N-1$ elements [7]. These sparse arrays structures can provide a large virtual array constructed from the difference co-array (DCA) of the two ULAs elements to enhance the DOA estimation. In passive sensing, the spatial correlation matrix depends on the measured data of the consecutive lags resulted from the DCA. Some elements may be missed (hole) in the DCA, while other elements may have frequently computed depending on the location of the elements in the two ULAs [8].

Recently, various coprime array configurations are proposed to overcome the holes problem, increment the number of consecutive lags, minimize the lags redundancy, provide a hole-free difference array, and enhance the DOA estimation accuracy[9]. For example, generalized coprime array (GCA) [10, 11] that modified the spacing between the elements by compressing one subarray and provide a proper 
displacement to the other one to enhance the DOFs. Shifted coprime array (SCA) $[12,13]$ that displaced one of the two subarrays, to fill the holes and generate more consecutive lags. Thinned coprime array (TCA) [14] reduced the lag frequency in the DCA using an identical number of physical elements. Unfolded coprime array (UFCA) [15] was presented to get higher DOFs with larger virtual aperture size by setting the two subarrays at the positive and negative sides of the $\mathrm{x}$-axis. All these arrays structures are based on a fixed array platform concept.

These array structures can be performed on a moving platform as in navigation, vehicle attached, and ship-based. The Synthetic array (SA) mounted on moving platform provides another form to extend the synthetic aperture size and improve the spatial resolution. SA uses the temporal signal coherence period (TCP) to span the array aperture [16-18]. The authors in [19] assumed that the TCP is $N / 2$ half the wavelength to provide a hole-free virtual array, which is unexpected for the source to stay constant for a long time specially for larger antenna aperture. In an environment with slow changing, the sensing environment such as source locations, angular steering, and source temporal composition can be considered unchanged within little noticeable time. [20].

The moved array can overcome the empty positions and provide distinct lags based on the actual element positions that sufficiently enhanced the DOFs and increase the central lags segment. The difference synthetic array is considered as the DCA of the actual array and the moved array with lag being shifted half the wavelength of the received sources to solve the holing issue in the DCA and increase the consecutive lags and also the unique lags [21, 22]. In fact, only the lags that are adjacent to the holes are helpful to fill the empty positions in the SA since the shifting of lags that create previously presented lags does not increase the number of DOFs. Authors in [23] proposed a moving array that can be applied to the coprime array and sparse uniform linear array (SULA), where data can be gathered at different array locations by shifting the array left and right, which give the chance to span the virtual array with more uniform central lags and more unique lags. In [21], the authors extended the two subarrays inter-element spacing to fill some holes and lengthened the number of DOFs. In [24], the authors proposed an improved unfolded coprime array (IUFCA) expose to movement to extend the number of uDOF. In [25], the authors proposed an array configuration of compressing elements distance to triple of one subarray, while extending the other one to obtain a completely fill array. The need for a fill free moved array with high number of DOFs still the main issue for applications such as ship-based platform since the signals with high frequency is exposed to highly absorption loss in underwater scenarios.

The objective of this paper is to enhance the number of uniform DOF and generate a fill unfolded coprime array configuration for moving platform by relocating some elements positions of the physical array and adjusting the inter-elements spacing to span and filled the virtual array. The array structure design is referred to as "Enhanced Unfolded Coprime Array" (EUFCA). The formal expressions for the uDOF and unique lags are derived. Thus, when the number of $\mathrm{uDOF}$ is increased the DOA estimation performance is enhanced effectively by detecting more source signals.

The paper is set such that: Section 2 demonstrates the data model performing on the moved array and the DCA of the synthetic array. Section 3 detailed the proposed EUFCA array structure along with formal expressions of uDOF. Section 4 presents the DOA estimation of moved array and computational complexity. Section 5 shows the lags generation comparisons and simulation results. Finally, the conclusion is given in Section 6.

Notations: we use upper-case (lower-case) bold characters to represent matrices (vectors). $[\cdot]^{T},[\cdot]^{*}$ and $[\cdot]^{H}$ stand for transpose, the conjugate and conjugate transpose of a vector or matrix respectively. $\operatorname{diag}($.$) and \operatorname{vec}(\cdot)$ mean a diagonal matrix and the vectorization operator. $E\{\cdot\}$ represents the expectation operator. $\mathrm{I}_{\mathrm{K}}$ indicates the identity matrix with size $K \times K$. $\otimes$ stands for the kronecker product. [.] denotes the floor function.

\section{Problem statement}

\subsection{Data model for coprime array}

An augmented coprime array (ACA) consists of two ULAs: $N$ and $2 M$ elements with $M d$ and $N d$ inter-elements distance respectively that is located in collinear as displayed in Fig. 1(a). The entire real elements in the ACA are $K=N+2 M-1$ since the reference element at zero position is shared by the two subarrays. The elements position of the actual array $\left(\mathbb{P}_{\text {org }}\right)$ can be denoted as:

$$
\begin{array}{r}
\mathbb{P}_{\text {org }}=\{\mathfrak{a} M d \mid \mathfrak{a}=0,1, \ldots,(N-1)\} \cup \\
\{\mathfrak{b} N d \mid \mathfrak{b}=0,1, \ldots,(2 M-1)\}
\end{array}
$$



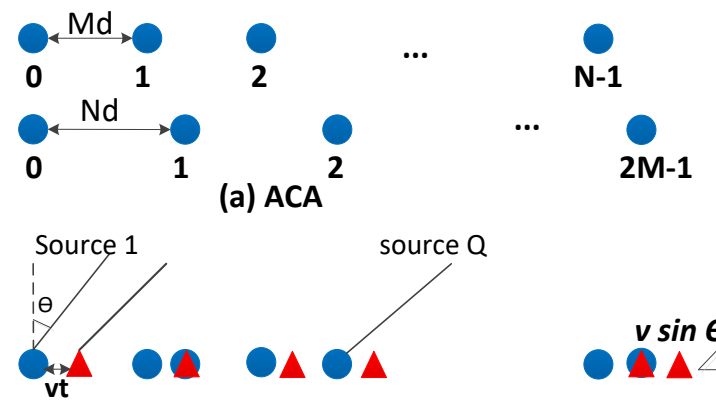

(b) Moved ACA

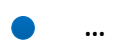

(c) UFCA

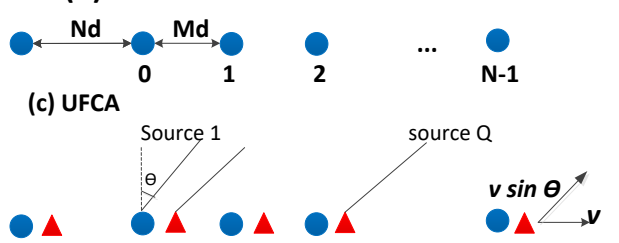

(d) Moved UFCA

Elements locations before motion $\Delta$ Elements locations after motion

Figure. 1 Array configuration for: (a) original ACA,

(b) moved ACA, (c) original UFCA, and (d) moved UFCA

where $d$ is the spacing between elements and its half the wavelength $(\lambda / 2)$. For a moving array at a constant velocity $v$, as shown in Fig. 1(b), the synthetic array $\left(\mathbb{P}_{\text {synth }}\right)$ is formed from the actual $\left(\mathbb{P}_{\text {org }}\right)$ and moved array $\left(\mathbb{P}_{\text {mov }}\right)$ can be expressed as.:

$$
\begin{aligned}
\mathbb{P}_{\text {mov }}= & \{\mathfrak{a} M d+1 \mid \mathfrak{a}=0,1, \ldots,(N-1)\} \cup \\
& \{\mathfrak{b} N d+1 \mid \mathfrak{b}=0,1, \ldots,(2 M-1)\} \\
& \mathbb{P}_{\text {synth }}=\mathbb{P}_{\text {org }} \cup \mathbb{P}_{\text {mov }}
\end{aligned}
$$

Suppose there is $Q$ far field narrowband uncorrelated sources from directions $\left\{\theta_{1}, \theta_{2}, \ldots, \theta_{q}\right\}$ are impinging in an antenna array of $K$ elements, the received signals $\boldsymbol{X}(t)$ at time $t$ can be expressed as:

$$
\begin{gathered}
\boldsymbol{X}(t)=\sum_{q=1}^{Q} \boldsymbol{a}\left(\theta_{q}\right) e^{\left(\frac{-j 2 \pi v t \sin \theta_{q}}{\lambda}\right)} S_{q}(t)+\boldsymbol{n}(t) \\
=\boldsymbol{A s}(t)+\boldsymbol{n}(t)
\end{gathered}
$$

where $\boldsymbol{A}=\left[\boldsymbol{a}\left(\theta_{1}\right), \boldsymbol{a}\left(\theta_{2}\right), \ldots, \boldsymbol{a}\left(\theta_{Q}\right)\right]$ and $\boldsymbol{a}\left(\theta_{q}\right)=$ $\left[1, e^{-j 2 \pi d \sin \left(\theta_{q}\right) / \lambda}, \ldots, e^{-j 2 \pi(K-1) d \sin \left(\theta_{q}\right) / \lambda}\right]^{T}$, is the $\mathrm{K} \times \mathrm{Q}$ directional matrix, $\boldsymbol{s}(t)=\left[s_{1}(t)\right.$ $\left.e^{-j 2 \pi v t \sin \theta_{q} / \lambda}, \ldots, s_{Q}(t) e^{-j 2 \pi v t \sin \theta_{q} / \lambda}\right]^{T}$ is the $Q \times 1$ source signal vector, and $\boldsymbol{n}(t)=\left[n_{1}(t)\right.$, $\left.\ldots, n_{K}(t)\right]^{T}$ is the $K \times 1$ noise vector which is an additive Gaussian white noise with variance $\sigma_{n}^{2}$ that is uniformly distributed. At time $t+\tau$, the received signal is expressed as:

$$
\begin{array}{r}
\boldsymbol{X}(t+\tau)= \\
\sum_{q=1}^{Q} \boldsymbol{a}\left(\theta_{q}\right) e^{\left(-\frac{j 2 \pi v t \sin \theta_{q}}{\lambda}\right)} e^{\left(-\frac{j 2 \pi v \tau \sin \theta_{q}}{\lambda}\right)} s_{q}(t+\tau)+ \\
\boldsymbol{n}(t+\tau)
\end{array}
$$

Where $\boldsymbol{B}=\left[\boldsymbol{b}\left(\theta_{1}\right), \boldsymbol{b}\left(\theta_{2}\right), \ldots \boldsymbol{b}\left(\theta_{Q}\right)\right]$ is the $K \times Q$ array direction matrix, $\boldsymbol{b}\left(\theta_{q}\right)=$ $\left[e^{-j 2 \pi v t \sin \left(\theta_{q}\right)}\right.$,

$\left.e^{-j 2 \pi\left(v t+d_{2}\right) \sin \left(\theta_{q}\right)}, \ldots, e^{-j 2 \pi\left(v t+d_{k}\right) \sin \left(\theta_{q}\right)}\right]^{T}$. Since the array moved half the wavelength $v t=$ $d=\lambda / 2$, then

$$
\boldsymbol{b}\left(\theta_{q}\right)=\left[e^{j 2 \pi d \sin \left(\theta_{q}\right) / \lambda}, \ldots, e^{j 2 \pi\left(d+d_{k}\right) \sin \left(\theta_{q}\right) / \lambda}\right]^{T}
$$

and

$$
\begin{gathered}
\boldsymbol{s}(t+\tau)=\left[s_{1}(t+\tau) e^{-j 2 \pi v t \sin \theta_{q} / \lambda}, s_{2}(t+\tau)\right. \\
\left.e^{-j 2 \pi v t \sin \theta_{2} / \lambda}, \ldots, s_{q}(t+\tau) e^{-j 2 \pi v t \sin \theta_{q} / \lambda}\right]^{T}
\end{gathered}
$$

For a narrowband signal with carrier frequency $(f), s_{q}(t+\tau)=s_{q}(t) e^{j 2 \pi f \tau}$. Then Eq. (5) can be rewritten as:

$$
\boldsymbol{X}(t+\tau)=e^{j 2 \pi f \tau} \boldsymbol{B} \boldsymbol{s}(t)+\boldsymbol{n}(t+\tau)
$$

Using the phase correcting factor $\left(e^{j 2 \pi f \tau}\right)$, a phase synchronized observed signal is determined as follows [19]:

$$
\widetilde{\boldsymbol{X}}(t+\tau)=\boldsymbol{X}(t+\tau) e^{-j 2 \pi f \tau}=\boldsymbol{B} \boldsymbol{s}(t)+\widetilde{\boldsymbol{n}}(t+\tau)
$$

where $\widetilde{\boldsymbol{n}}(t+\tau)=e^{j 2 \pi f \tau} \boldsymbol{n}(t+\tau)$. By combining Eqs. (4) and (9), the received signal of the synthetic arrays is denoted as:

$$
\boldsymbol{z}(t)=\left[\begin{array}{c}
\boldsymbol{X}(t) \\
\widetilde{\boldsymbol{X}}(t+\tau)
\end{array}\right]=\boldsymbol{A}_{\text {synth }} \boldsymbol{s}(t)+\left[\begin{array}{c}
\boldsymbol{n}(t) \\
\widetilde{\boldsymbol{n}}(t+\tau)
\end{array}\right]
$$

where $\boldsymbol{A}_{\text {synth }}=\left[\boldsymbol{A}^{T}, \boldsymbol{B}^{T}\right]^{T}$, is the steering matrix of the synthetic array.

\subsection{Synthetic difference co-array}

Unfolded coprime array (UFCA) have two subarrays with $N$ and $2 M$ elements with $M d$ and $N d$ spacing between the inter-elements successively as displayed in Fig. 1(c). $N, M$ are integers, $N>M$ and the $\operatorname{GCD}(N, M)=1$. The $\mathrm{N}$-subarray is set at the positive side of the $\mathrm{X}$-axis within range $\{0: M:(N-$ 
1) $M d\}$, while the M-subarray is set at the negative side of the $\mathrm{x}$-axis within range $-\{0: N:(2 M-$ 1) $N d\}$. The two subarrays are arranged in a collinear manner. The physical elements positions before motion can be denoted as:

$$
\begin{aligned}
\mathbb{P}_{U F C A}=\{\mathfrak{a} M d \mid \mathfrak{a} & =0,1, \ldots,(N-1)\} \cup \\
\{-\mathfrak{b} N d \mid \mathfrak{b} & =0,1, \ldots,(2 M-1)\}
\end{aligned}
$$

After moving a half of the wavelength, the two subarrays positioned can be given as:

$$
\begin{array}{r}
\mathbb{P}_{U F C A-\text { moved }}=\{\mathfrak{a} M d+d \mid \mathfrak{a}=0,1, \ldots,(N-1)\} \cup \\
\{-\mathfrak{b} N d+d \mid \mathfrak{b}=0,1, \ldots,(2 M-1)\} \quad(12)
\end{array}
$$

The synthetic array is formed from the actual and shifted arrays, so the DCA is constructed from the cross-differences of all the lags in the actual and shifted arrays since the self-difference lags are part of the cross-difference. So the cross difference can be expressed as:

$$
\mathbb{D}=\mathbb{D}_{12} \cup \mathbb{D}_{13} \cup \mathbb{D}_{14} \cup \mathbb{D}_{23} \cup \mathbb{D}_{24} \cup \mathbb{D}_{34}
$$

where the subscripts 1 and 2 points refer to the two actual subarrays, and the subscripts 3 and 4 points refer to the two shifted subarrays. $\mathbb{D}_{12}$ is the crossdifference of the lags between the two subarrays $(1,2)$ of the actual array, $\mathbb{D}_{13}$ and $\mathbb{D}_{14}$ refer to the cross-difference of the lags between the actual subarray 1 and the shifted subarray 3 and 4 respectively. $\mathbb{D}_{23}$ and $\mathbb{D}_{24}$ refer to the crossdifference between the actual subarray 2 and the shifted subarray 3 and 4 respectively, and $\mathbb{D}_{34}$ is the cross-difference of the lags between the subarray 3 and the subarray 4 of the shifted array which is obtained as follows:

$$
\begin{gathered}
\mathbb{D}_{12}=\left\{M a_{1}-N a_{2}\right\} \cup\left\{N a_{2}-M a_{1}\right\} \\
\mathbb{D}_{13}=\left\{M a_{1}-N a_{3}-1\right\} \cup\left\{N a_{3}-M a_{1}+1\right\} \\
\mathbb{D}_{14}=\left\{M a_{1}-N a_{4}-1\right\} \cup\left\{N a_{4}-M a_{1}+1\right\} \\
\mathbb{D}_{23}=\left\{N a_{2}-M a_{3}-1\right\} \cup\left\{M a_{3}-N a_{2}+1\right\} \\
\mathbb{D}_{24}=\left\{N a_{2}-M a_{4}-1\right\} \cup\left\{M a_{4}-M a_{2}+1\right\} \\
\mathbb{D}_{34}=\left\{M a_{3}-N a_{4}\right\} \cup\left\{N a_{4}-M a_{3}\right\}
\end{gathered}
$$

Where $0 \leq a_{1}, a_{3} \leq N-1,0 \leq a_{2}, a_{4} \leq 2 M-$ 1 , it is verified in [23] and [20] that $\mathbb{D}_{12}=\mathbb{D}_{12} \cup$ $\mathbb{D}_{34}$, and $\mathbb{D}_{14} \cup \mathbb{D}_{23}=\mathbb{D}_{13} \cup \mathbb{D}_{24} \cup \mathbb{D}_{14} \cup \mathbb{D}_{23}$, so the synthetic array can be rewritten as:

$$
\mathbb{D}_{\text {synth }}=\mathbb{D}_{12} \cup \mathbb{D}_{14} \cup \mathbb{D}_{23}
$$

$\mathbb{D}_{14}$ and $\mathbb{D}_{23}$ might be considered as the DCA of the actual array being shifted by $\lambda / 2 d$ once to the left and once to the right which can demonstrate the increasing of the lags as the array moved.

For an example of moved array, Fig. 2(a) shows UFCA with $M=3$, and $N=5$. The actual DCA can generate a consecutive lags within the range $\{ \pm 8, \pm 9, \ldots, \pm 29\}$ and the number of uDOF is 22 . The holes are located at the central of the actual DCA in the set $\{ \pm 1, \pm 2, \pm 4, \pm 7\}$. After array moving within $(\lambda / 2) d$, all the holes are filled since the synthetic DCA can fill two adjacent holes by the shifting the lag to the right and to the left.

Fig. 2(b) displays UFCA with $M=4$, and $N=$ 5 . The actual DCA can generate a consecutive lags within the range $\{ \pm 12, \pm 13, \ldots, \pm 39\}$ and the number of $\mathrm{uDOF}$ is 28 . The holes are located at the

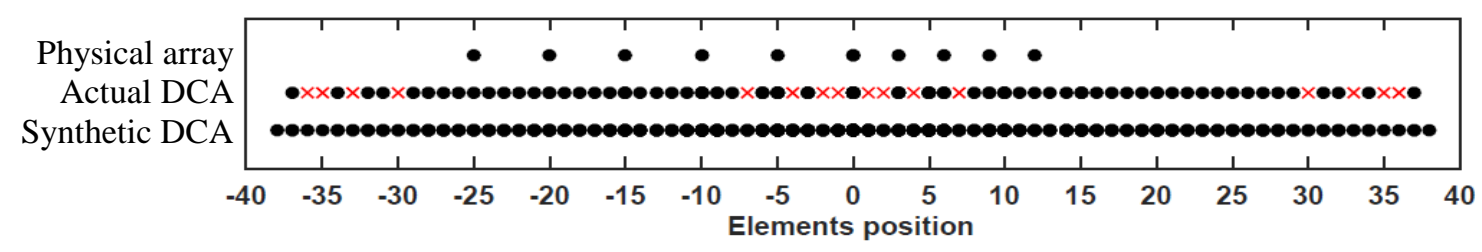

(a)

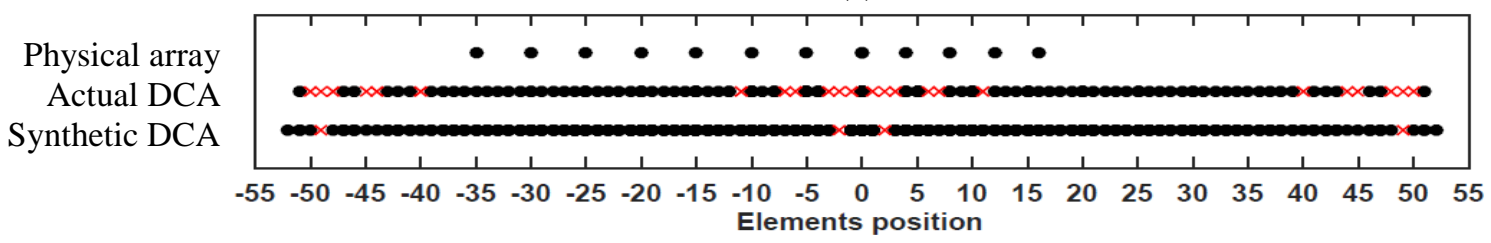

(b)

Figure. 2 The actual and synthetic DCA of unfolded coprime array configuration when (a) $\mathrm{M}=3, \mathrm{~N}=5$ and (b) $\mathrm{M}=4, \mathrm{~N}=5$ 
central of the actual DCA in the set $\{ \pm 1, \pm 2, \pm 3, \pm 4$ $\pm 7, \pm 8, \pm 11\}$. After array moving, not all the holes are filled in the synthetic DCA, there is still a hole at the center of the synthetic DCA, due to that the synthetic DCA is able to fill two neighbor holes only, when the number of consecutive holes more than two, the holes can't be filled.

\section{Proposed moving platform array}

As mentioned in the previous section, motion array can fill the central hole when $M \leq 3$, but when $M \geq 4$, and $N=\gamma M-1, \gamma \geq 3$, still there is empty positions at the center of the synthetic DCA which impact the resolution of DOA estimation process. The proposed EUFCA moved array structure design can be developed considering the UFCA by reallocating the last elements positions of $\mathrm{M}$ subarray. We suggest two designs for two different situations to get a full filled DCA synthetic array according to the $(\mathrm{M}, \mathrm{N})$ pair value.

Case1: By modified the positions of $M_{t}=$ $\lfloor M / 2\rfloor-1$ physical elements of M-subarray and appended them to $\mathrm{N}$-subarray at positions $(N-$ 1) $M+3 a_{0}, a_{0} \in[1,\lfloor M / 2\rfloor-1]$, as shown in Fig. $3(\mathrm{a})$. The elements positions in this case can be donated as:

$$
\begin{gathered}
\mathbb{P}_{E U F C A-1}=\{\mathfrak{a} M d \mid \mathfrak{a}=0,1, \ldots,(N-1)\} \cup \\
\left\{-\mathfrak{b} N d \mid \mathfrak{b}=0,1, \ldots,\left(2 M-M_{t}-1\right)\right\} \cup \\
\left\{(N-1) M+3 a_{0}, a_{0} \mid=1, \ldots, M_{t}\right\}
\end{gathered}
$$

All the holes at the center of the DCA synthetic array are filled with lags expansion which provides a hole free virtual array. This situation is suitable only when $N=M+1$, and $M \geq 4$. The number of uDOF generated can expressed as follows:

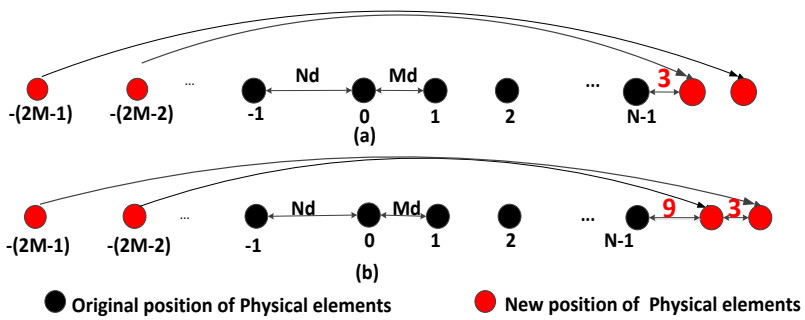

Figure. 3 proposed EUFCA configuration

$$
u D O F_{E U F C A-1}=3 M N+\left\lfloor\frac{M}{2}\right\rfloor(3-N)-M-1
$$

Here the number of $\mathrm{uDOF}$ is the same as the number of unique lags and the virtual array aperture size since it's a hole free array.

Case 2: for $M \geq 4$, with different pairs of $(M, N), M_{t}=M-1$ physical elements positions of M-subarray are reallocated and appended to the $\mathrm{N}$ subarray at positions $9+(N-1) M+3 a_{0}, a_{0} \in$ $[1, M-1]$ as shown in Fig. 3(b), The elements positions in this case can be donated as:

$$
\begin{gathered}
\mathbb{P}_{\text {EUFCA-2 }}=\{\mathfrak{a} M d \mid \mathfrak{a}=0,1, \ldots,(N-1)\} \cup \\
\left\{-\mathfrak{b} N d \mid \mathfrak{b}=0,1, \ldots,\left(2 M-M_{t}-1\right)\right\} \\
\cup\left\{9+(N-1) M+3 a_{0}, a_{0} \mid=1, \ldots, M_{t}\right\}
\end{gathered}
$$

The DCA of synthetic array has a full array with no empty positions so we get a high central consecutive lag with larger aperture size. In this case the number of uDOF generated can expressed as follows:

$$
u D O F_{E U F C A-2}=2 M N+2 M+8
$$

For example, Fig. 4 shows the two proposed EUFCA with $M=5, N=6$, and $N=M+1$, where

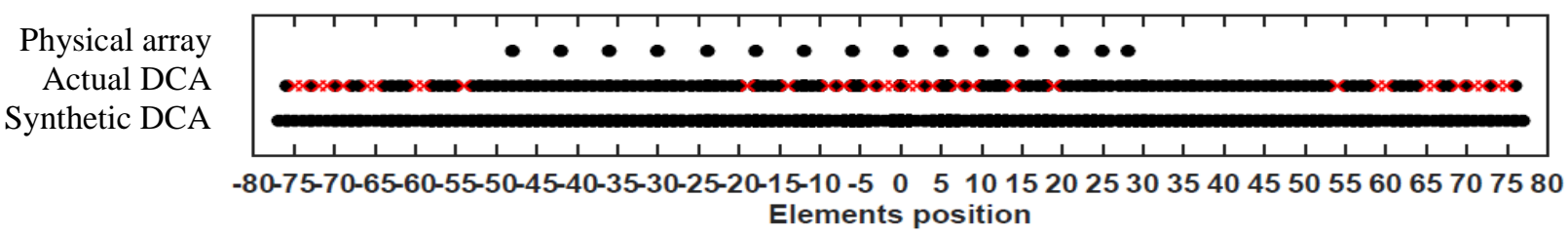

(a)

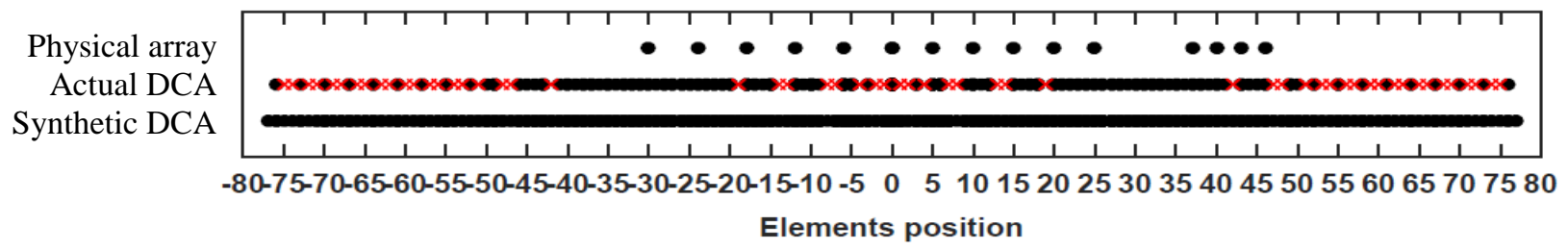

(b)

Figure. 4 EUFCA configuration when $\mathrm{M}=5, \mathrm{~N}=6$ (a) the first case design and (b) the second case design 
Fig. 4(a) and (b) are for using the first case and the second case respectively. The figure shows the physical element position, the DCA of the actual array before motion, and the DCA of the synthetic array after shifting the actual array. In the two cases, the EUFCA configuration array can create a hole free array by filling all the holes in the synthetic array and increase the number of the central lags to 153 and 155 for the two cases respectively. We have noticed that when $M \geq 8$, the first case can generate more consecutive lags than the first case.

Another example for the proposed EUFCA array configuration when $N=2 M-1$, and $M=4, N=$ 7 is shown in Fig. 5 for both first and second cases design. The figure shows the physical element position, and the DCA of the actual and synthetic arrays.

In the first cases, the EUFCA configuration array can have a hole in the DCA of the synthetic array with 127 consecutive lags, while in the second case; we get a full filled DCA with 143 consecutive lags. The second case can perform better for different values of $(M, N)$ pairs.

\section{DOA estimation using SS-MUSIC}

\subsection{DOA estimation}

For DOA estimation, the spatial co-array MUSIC is performed on the consecutive lags of the EUFCA after vectorizing the correlation matrix of $z(t)$ [26]. From Eq. (8), the correlation matrix is expressed as:

$$
R=E\left\{z(t) z^{H}(t)\right\}=A_{\text {synth }} R_{s} A_{\text {synth }}^{H}+\sigma_{n}^{2} I
$$

Where $R_{s}=E\left\{s(t) s^{H}(t)=\operatorname{diag}\left(\sigma_{1}, \sigma_{2}, \ldots, \sigma_{Q}\right)\right.$ is the source signal correlation matrix, $\sigma_{\mathrm{n}}^{2}$ is the noise variance and $I$ is the identity matrix. Vectorizing $\mathrm{R}$, the following is obtained:

$$
y=\operatorname{vec}(R)=\tilde{A} P+\sigma_{n}^{2} i
$$

Where $\tilde{A}=\left[\tilde{a}\left(\theta_{1}\right), \tilde{a}\left(\theta_{2}\right), \ldots \tilde{a}\left(\theta_{Q}\right)\right], \tilde{a}\left(\theta_{q}\right)=$ $a_{s}{ }^{*}\left(\theta_{q}\right) \otimes a_{s}\left(\theta_{q}\right)$ is the data matrix of the DCA that have only the consecutive lags after removing the repeated lags. $P$ is the source signals. $\otimes$ is the Kronecker product. Then the correlation matrix for the consecutive lags is determined as follows:

$$
R R=E\left\{y(t) y^{H}(t)\right\}
$$

Then, the MUSIC method is performed to estimate the uncorrelated signals with high resolution.

\subsection{Computational complexity}

The computational complexity derived from the computations of the correlation matrix and the reconstruction of the virtual array. For coaaryMUSIC, the complexity is $O\left\{\left(J K^{2}\right)+2 U^{3}+\right.$ $\left.t_{s}(2 U(U-Q)+U)\right\}$, where $J$ represents the number of the snapshots, $K$ is the number of the physical elements in an antenna array, $U$ is the number of the consecutive elements in the virtual array and $t_{s}$ is the search time. Fig. 6 shows the comparative computational complexity of the original and synthetic array for UFCA [15], IUFCA [24] and EUFCA using different number of physical elements $(12,14,18)$. It can be shown that the proposed synthetic EUCFA has the highest

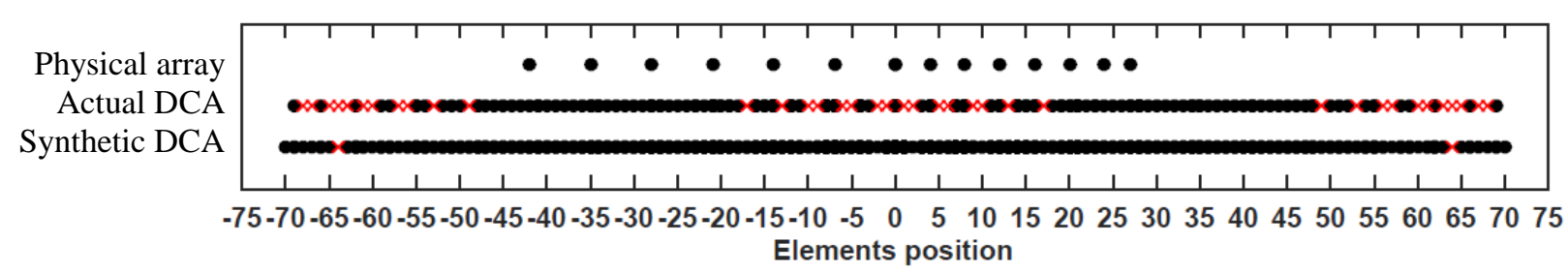

(a)

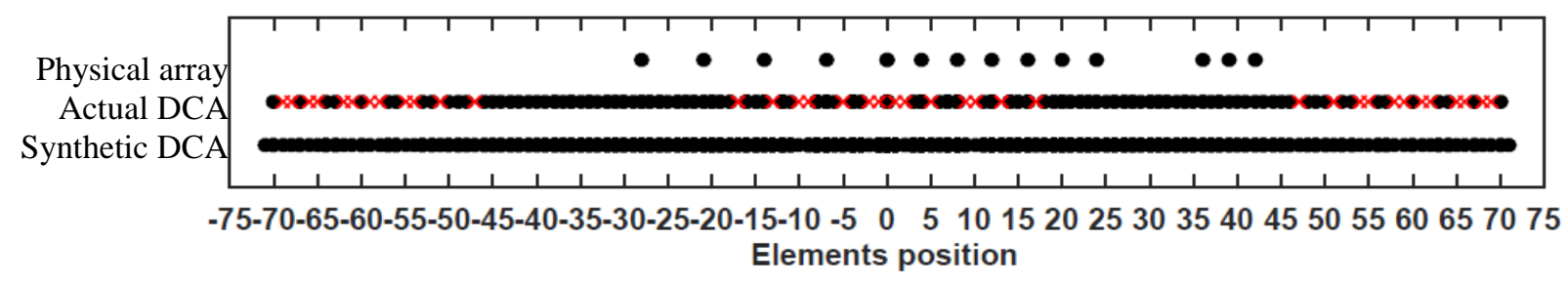

(b)

Figure. 5 EUFCA configuration when $\mathrm{M}=4, \mathrm{~N}=7$ (a) the first case design and (b) the second case 
Table. 1 comparison for various array types before and after motion considering the uDOF and NCLR

\begin{tabular}{|c|c|c|c|c|c|c|c|c|c|c|c|c|c|c|c|c|}
\hline \multirow[b]{2}{*}{$\mathbf{M}, \mathbf{N}$} & \multirow[b]{2}{*}{$\begin{array}{l}\text { number } \\
\text { of } \\
\text { elements }\end{array}$} & \multicolumn{5}{|c|}{ uDOF before array motion } & \multicolumn{5}{|c|}{ uDOF after arrav motion } & \multicolumn{5}{|c|}{ NCLR } \\
\hline & & $\begin{array}{c}\text { ACA } \\
{[6]}\end{array}$ & $\begin{array}{c}\text { UFC } \\
\text { A } \\
{[15]}\end{array}$ & $\begin{array}{l}\text { IUF } \\
\text { CA } \\
\text { [24] }\end{array}$ & $\begin{array}{c}\text { EUF } \\
\text { CA- } \\
\text { case } \\
1 \\
\end{array}$ & \begin{tabular}{|c} 
EUF \\
CA- \\
case \\
2 \\
\end{tabular} & $\begin{array}{c}\text { ACA } \\
{[20]}\end{array}$ & $\begin{array}{c}\text { UFC } \\
\text { A }\end{array}$ & $\begin{array}{c}\text { IUF } \\
\text { CA } \\
{[24]}\end{array}$ & \begin{tabular}{|c} 
EUF \\
CA- \\
case \\
1 \\
\end{tabular} & \begin{tabular}{|c} 
EUF \\
CA- \\
case \\
2 \\
\end{tabular} & $\begin{array}{c}\text { ACA } \\
{[20]}\end{array}$ & $\begin{array}{c}\text { UFC } \\
\mathbf{A}\end{array}$ & $\begin{array}{c}\text { IUF } \\
\text { CA } \\
\text { [24] }\end{array}$ & \begin{tabular}{|c} 
EUF \\
CA- \\
case \\
1 \\
\end{tabular} & $\begin{array}{c}\text { EUF } \\
\text { CA- } \\
\text { case } \\
2 \\
\end{array}$ \\
\hline 4,5 & 12 & 47 & 28 & 28 & 28 & 13 & 65 & 46 & 87 & 101 & 111 & 1.38 & 1.64 & 3.11 & 3.61 & 8.54 \\
\hline 4,7 & 14 & 63 & 38 & 32 & 31 & 29 & 93 & 68 & 127 & 127 & 143 & 1.48 & 1.79 & 3.97 & 4.10 & 4.93 \\
\hline 5,6 & 15 & 69 & 40 & 38 & 38 & 22 & 91 & 62 & 129 & 155 & 155 & 1.32 & 1.55 & 3.39 & 4.08 & 7.05 \\
\hline 4,9 & 16 & 79 & 48 & 39 & 48 & 32 & 113 & 82 & 159 & 167 & 175 & 1.43 & 1.71 & 4.08 & 3.48 & 5.47 \\
\hline 5,8 & 17 & 89 & 52 & 44 & 48 & 48 & 137 & 100 & 191 & 191 & 195 & 1.54 & 1.92 & 4.34 & 3.98 & 4.06 \\
\hline 5,9 & 18 & 99 & 58 & 49 & 49 & 37 & 137 & 96 & 199 & 199 & 215 & 1.38 & 1.66 & 4.06 & 4.06 & 5.81 \\
\hline 6,7 & 18 & 95 & 54 & 40 & 46 & 19 & 121 & 80 & 165 & 213 & 207 & \begin{tabular}{|l}
1.27 \\
\end{tabular} & \begin{tabular}{|l}
1.48 \\
\end{tabular} & 4.13 & 4.63 & 10.89 \\
\hline 5,11 & 20 & 119 & 70 & 59 & 57 & 37 & \begin{tabular}{|l|}
161 \\
\end{tabular} & 132 & 239 & \begin{tabular}{|l|}
249 \\
\end{tabular} & 255 & \begin{tabular}{|l}
1.35 \\
\end{tabular} & \begin{tabular}{|l}
1.89 \\
\end{tabular} & 4.05 & 4.37 & 6.89 \\
\hline 7,8 & 21 & 125 & 70 & 54 & 62 & 50 & 155 & 107 & 221 & \begin{tabular}{|l|}
289 \\
\end{tabular} & 267 & \begin{tabular}{|l}
1.24 \\
\end{tabular} & 1.53 & 4.09 & 4.66 & 5.34 \\
\hline 5,12 & 21 & 129 & 76 & 68 & 76 & 52 & 199 & 156 & 285 & 287 & 275 & \begin{tabular}{|l}
1.54 \\
\end{tabular} & 2.05 & 4.19 & 3.78 & 5.29 \\
\hline 6,11 & 22 & 143 & 82 & 60 & 71 & 27 & 189 & 128 & 265 & 289 & 303 & 1.32 & 1.56 & 4.42 & 4.07 & 11.22 \\
\hline 7,9 & 22 & 139 & 78 & 60 & 60 & 51 & \begin{tabular}{|l|}
209 \\
\end{tabular} & 148 & 285 & 325 & 295 & \begin{tabular}{|l}
1.50 \\
\end{tabular} & \begin{tabular}{|l|}
1.90 \\
\end{tabular} & 4.75 & 5.42 & 5.78 \\
\hline
\end{tabular}

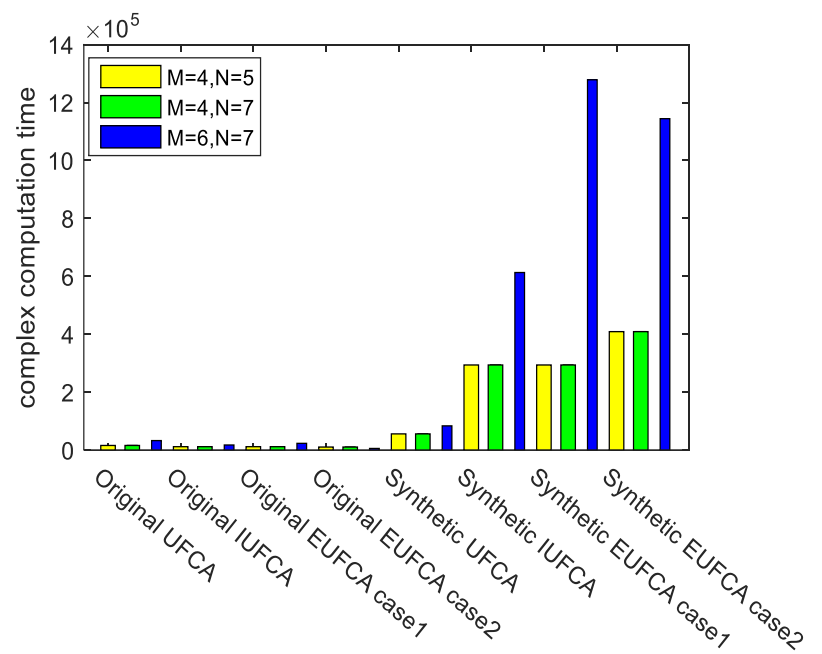

Figure. 6 Computational complexity comparison

computational complexity compared to the other synthetic arrays since it has the highest number of the $\mathrm{uDOF}$ and the computational complexity is increased relative to the number of physical number of elements.

\section{Simulation result}

The performance of EUFCA array design is evaluated considering the number of the lags being created and the spectrum estimation before and after array motion with other array types such ACA [6], moved ACA [20], UFCA [15], and IUFCA [24].

\subsection{Lag generation comparison of different array types before and after array motion}

Lag generation of various array types are compared according to the length of the uDOF that established from the DCA and the ratio of the number of consecutive lags (NCLR) before and after array motion. The comparison is performed using the same number of elements in each array design.

Table 1 shows different $(M, N)$ pairs that are compared different array types such as ACA [6], UFCA [15], IUFCA [24] and the proposed array configuration (EUFCA) before and after array motion. It can be shown that UFCA array has the less number of $\mathrm{uDOF}$ before and after array motion, that means the less of the number DOFs that can resolved. It is obvious that the proposed array EUFCA can perform better after array motion, since it can generate the largest number of uDOF than other array types.

\subsection{Spectrum estimation for different array types before and after array motion}

The spectrum estimation of UFCA [15], IUFCA [24] and EUFCA arrays before and after array motion are presented in this subsection using different values of $\mathrm{Q}$ according the number of $\mathrm{uDOF}$ that these arrays can resolved. The same number of the elements are set to all the above arrays, where $M=4, N=5$. The sources directions are within range [-70:70], signal to noise ratio $(\mathrm{SNR}=0 \mathrm{~dB})$ and snapshots $(\mathrm{T}=1000)$. Fig. 7 shows the spectrum 
estimation before array motion, for Fig. 7 (a) UFCA, (b) IUFCA and (c) EUFCA-case 1, the number of sources is set to 11 source since these arrays can resolve 11 sources due to the number of uDOFs that

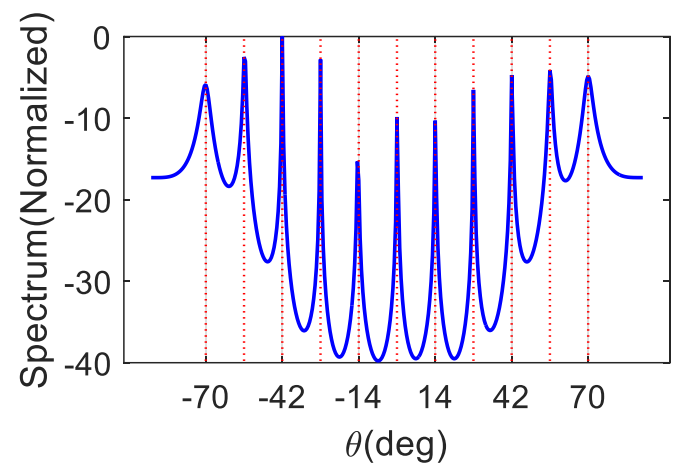

(a)

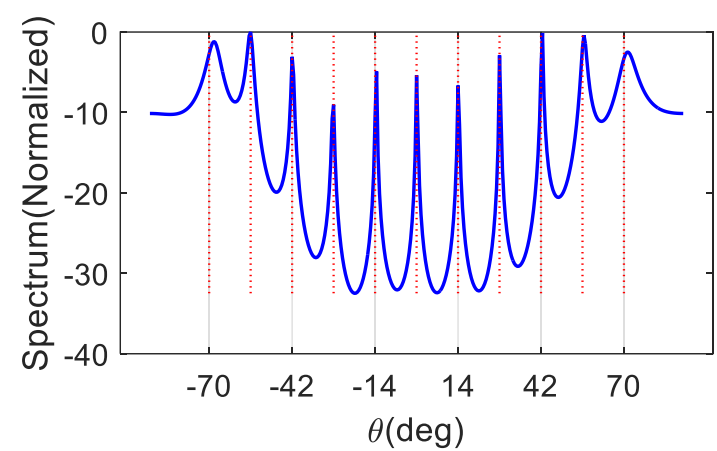

(c) they can generate. While in Fig. 6 (d) EUFCA-case2 the number of sources is set to 6 sources, due to the number of uDOFs which is 7 . It can be shown that all the sources can be resolved accurately since they

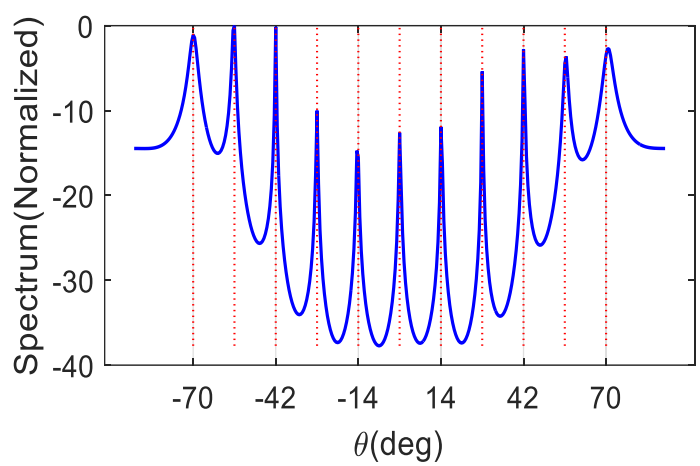

(b)

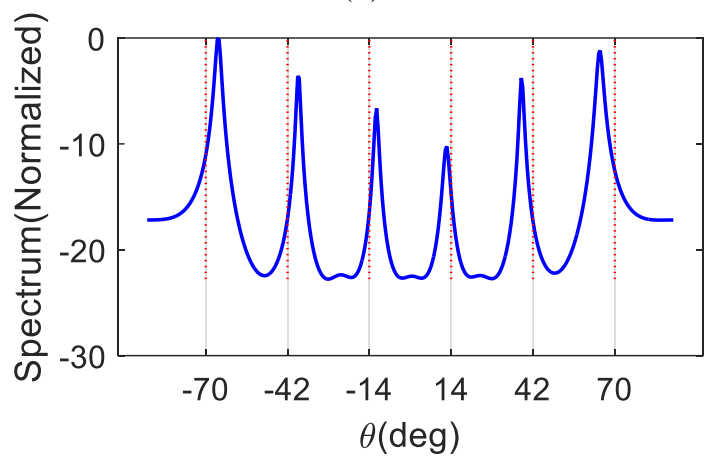

(d)

Figure. 7 Spectrum estimation before array motion: (a) UFCA, (b)IUFCA, (c)EUFCA-case1, and (d) EUFCA case2 with $M=4, N=5, \mathrm{Q}=11$, Snapshots $=1000 ; \mathrm{SNR}=0 \mathrm{~dB}$

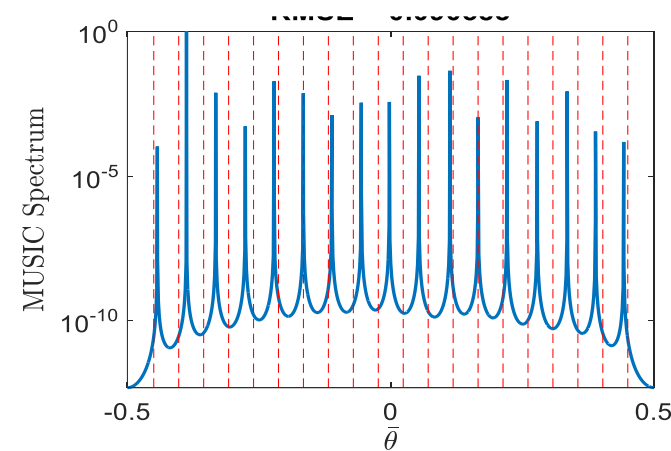

(a)

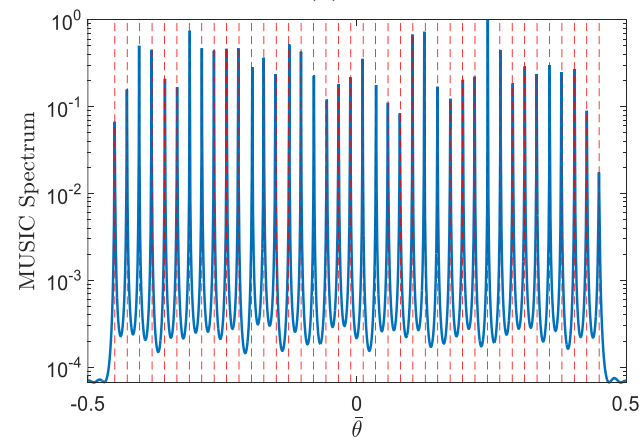

(c)

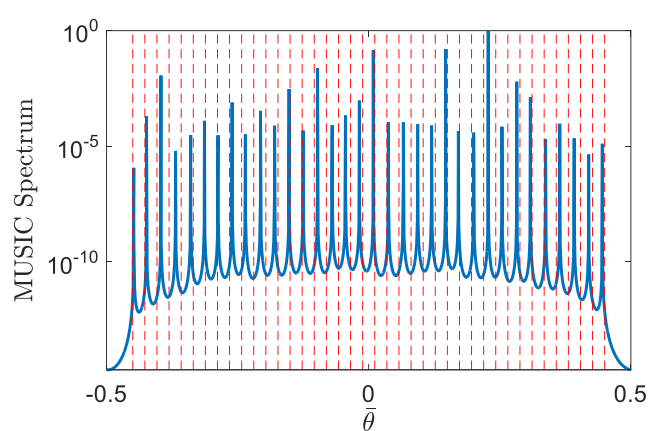

(b)

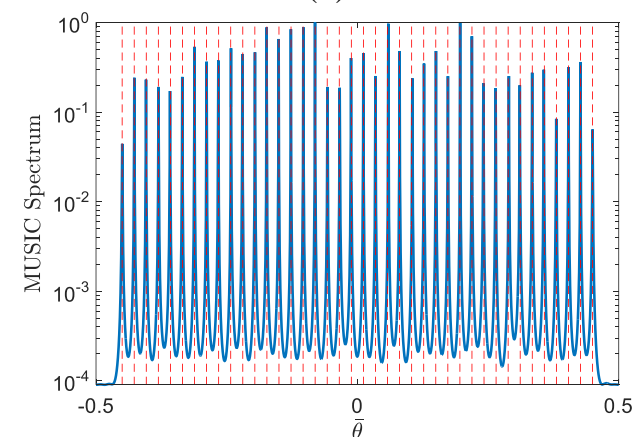

(d)

Figure. 8 Spectrum estimation after array motion: (a) $M=4, N=5, Q=35$ for the first case design and (b) $M=4, N=7, Q=55$ for the second case design 
are less than the number of uDOFs. Fig. 8 shows the spectrum estimation after array motion for (a)UFCA, (b)IUFCA, (c) EUFCA-case1 and (d)EUFCA-case2. The number of sources in Fig. 8(a) is set to 17, while in Fig. 8(b) is set to 35 due to the number of uDOFs in these array structure which is 24 and 44 respectively while the number of sources is 40 for both EUFCA cases. It can be noticed that all the sources have been correctly estimated for all the scenarios but the proposed EUCA can estimated more sources than other arrays due to the large number of the uDOFs that can generate.

\subsection{RMSE performance}

RMSE is a metric used to evaluate the DOA estimation. The RMSE is defined as follows:

$$
R M S E=\sqrt{1 / Q M_{c} \sum_{c=1}^{M_{c}} \sum_{q=1}^{Q}\left(\theta_{q}-\hat{\theta}_{q, c}\right)^{2}}
$$

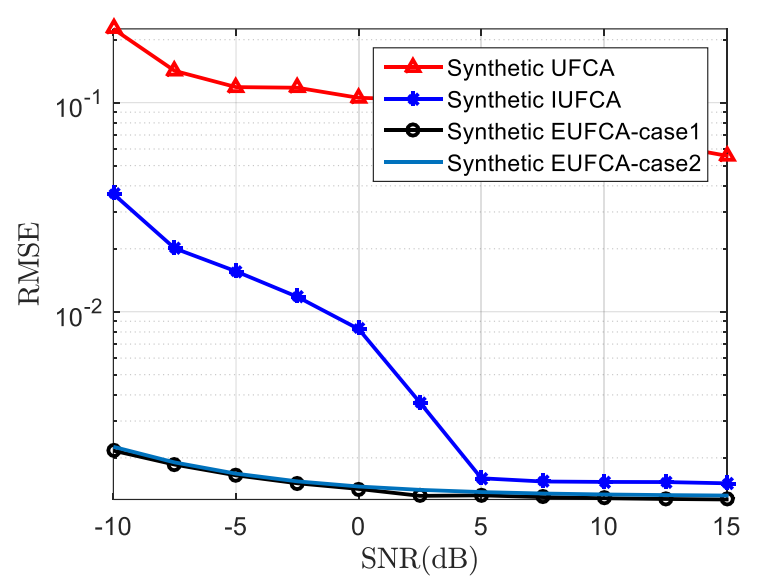

(a)

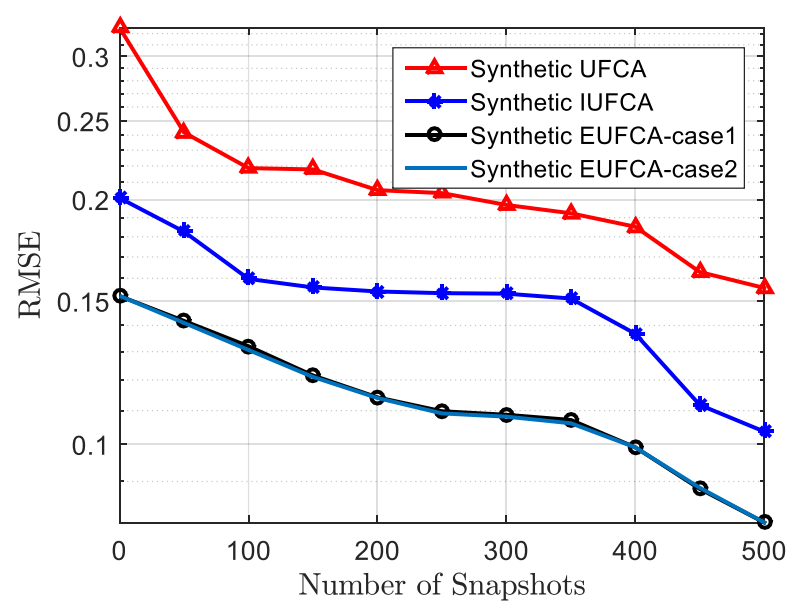

(b)

Figure. 9 RMSE performance of EUFCA with other array designs: (a) with respect to SNR variation and (b) with respect to different number of snapshots where $\theta_{q}$ is the true DOA of qth signals, $\hat{\theta}_{q, c}$ is the estimated DOA of cth trail, and $\mathrm{M}_{\mathrm{c}}$ is the total number of the trials. Fig. 9 shows the RMSE against SNR and the number of snapshots for different array types. For the two scenarios, the total number of elements is 12, the sources are 13. In Fig. 9(a), SNR varies from $-10 \mathrm{~dB}$ to $15 \mathrm{~dB}$, and the number of snapshots is set to 500. The synthetic EUFCA for the two cases have the same performance, and better than other arrays types at low SNR. This is because the two cases can generate approximately the same length of the consecutive lags and larger than other arrays. Fig. 9(b) shows the RMSE against the number of snapshots, where the snapshots range is from 20 to 500 , SNR is $10 \mathrm{~dB}$. Again, the EUFCA for the two cases has the same performance for the same reason of the above; the synthetic EUFCA has better performance than other array types.

\section{Conclusion}

In an environment such as the ship-based with slow motion, the source location can be supposed to be fixed for a small time. Using a sparse array in the receiver with a small motion, the original position and shifted position of the elements in the array can provide a large number of DOFs than being in one fixed position. In this paper, EUFCA is suggested to tackle the leakages of the central consecutive lags in the difference co-array. By relocating the position of the physical elements in the actual array and perform an array motion half the wavelength, we address this leakage by filling the holes and enlarge the uDOF. Hence the DOA estimation process is enhanced since more sources can be resolved.

\section{Conflicts of Interest}

The authors declare no conflicts of interest

\section{Author Contributions}

Both authors contributed to the work in the paper (Methodology, design, implementation, analysis, writing - original draft preparation, and writing-review and editing)

\section{References}

[1] M. Muhammad, M. Li, Q. H. Abbasi, C. Goh, and M. Imran, "Performance Evaluation for Direction of Arrival Estimation Using 4Element Linear Array", In: Proc. of 13th European Conference on Antennas and Propagation, 2019. 
[2] F. A. Salman and B. M. Sabbar, "Estimation of Coherent Signal on Modified Coprime Array", In: Proc. of 16th International Middle Eastern Simulation and Modelling Conference, pp. 6570, 2020.

[3] A. Moffet, "Minimum-Redundancy Linear Arrays", IEEE Trans. Antennas Propagation, Vol. 16, No. 2, pp. 172-175, 1968.

[4] D. A. Linebarger, I. H. Sudborough, and I. G. Tollis, "Difference Bases and Sparse Sensor Arrays", IEEE Trans. Inf. Theory, Vol. 39, No. 2, pp. 716-712, 1993.

[5] P. Pal and P. P. Vaidyanathan, "Nested Arrays: A Novel Approach to Array Processing with Enhanced Degrees of Freedom", IEEE Trans. Signal Process., Vol. 58, No. 8, pp. 4167-4181, 2010.

[6] P. P. Vaidyanathan and P. Pal, "Sparse Sensing with Co-prime Samplers and Arrays", IEEE Trans. Signal Process., Vol. 59, No. 2, pp. 573586, 2011.

[7] Y. D. Zhang, S. Qin, and M. G. Amin, "DOA Estimation Exploiting Coprime Arrays with Sparse Sensor Spacing”, In: Proc. of IEEE International Conference on Acoustics, Speech and Signal Processing, 2014.

[8] A. Ahmed, Y. D. Zhang, and J. Zhang, "Coprime Array Design with Minimum Lag Redundancy", In: Proc. of IEEE International Conference on Acoustics, Speech and Signal Processing, 2019.

[9] F. A. Salman and B. M.Sabbar, "Coprime Array Parameters Optimization for DOA estimation", Iraqi Journal of Information and Communications Technology, Vol. 4, Issue 2, 2021.

[10] S. Qin, Y. D. Zhang, and M. G. Amin, "Generalized Coprime Array Configurations for Direction-of-arrival Estimation", IEEE Trans. Signal Process., Vol. 63, No. 6, pp. 1377-1390, 2015.

[11] K. Shabir, T. H. A. Mahmud, R. Zheng, and Z. Ye, "Generalized Super-resolution DOA Estimation Array Configurations Design Exploiting Sparsity in Coprime Arrays", Circuits, Syst. Signal Process., Vol. 38, No. 6, pp. 4723-4738, 2019.

[12] S. M. Alamoudi, M. A. Aldhaheri, S. A. Alawsh, and A. H. Muqaibel, "Sparse DOA Estimation Based on a Shifted Coprime Array Configuration", In: Proc. of Mediterranean Microwave Symposium, 2016.

[13] A. Mohammed, A. Shaalan, and X. Yu, "DOA Estimation Based on the Optimized Coprime
Array Configuration", IEEE Access, Vol. 7, pp. 38789-38797, 2019.

[14] A. Raza, W. Liu, and Q. Shen, "Thinned Coprime Arrays for DOA Estimation", In: Proc. of 25th Eur. Signal Process. Conf., pp. 395-399, 2017.

[15] J. Li and X. Zhang, "Direction of Arrival Estimation of Quasi-Stationary Signals Using Unfolded Coprime Array", IEEE Access, Vol. 5, pp. 6538-6545, 2017.

[16] N. Wachowski and M. R. A. Sadjadi, "A New Synthetic Aperture Sonar Processing Method Using Coherence Analysis", IEEE J. Ocean. Eng., Vol. 36, Issue 5, pp. 665-678, 2011.

[17] Z. Shi, X. Zhang, and Y. Sun, "DOA Estimation with Synthetic Nested Array Using Moving Sensor", In: Proc. of 2nd International Conference on Machinary, Electronics, and Control Simulation, Advances in Engineering Research, Vol. 138, pp. 749-755, 2017.

[18] S. Zhan and Z. Xiaofei, "DOA Estimation Algorithm with Non-unifrom Motion Synthetic Linear Array", Transcation of Nanjing University of Aeronautics and Astronautics, Vol. 35, No. 5, pp. 75-80, 2018.

[19] J. Ramirez and J. L. Krolik, "Synthetic Aperture Processing for Passive Co-prime Linear Sensor Arrays", Digital Signal Process. A Rev. Journal, Vol. 61, Issue C, pp. 62-75, 2017.

[20] G. Qin, M. G. Amin, and Y. D. Zhang, "Analysis of Coprime Arrays on Moving Platform", In: Proc. of IEEE International Conference on Acoustics, Speech and Signal Processing, 2019.

[21] X. Yang, Y. Wang, and P. Charge, "Improved Coprime Linear Array Configuration for Moving Platform in DOA Estimation", IEEE Commun. Lett., Vol. 25, No. 2, pp. 470-473, 2021.

[22] G. Qin and M. G. Amin, "Optimum sparse array configuration for DOA estimation on moving platforms", In: Proc. of IEEE Radar Conference, 2020.

[23] G. Qin, M. G. Amin, and Y. D. Zhang, "DOA estimation exploiting sparse array motions", IEEE Transactions on Signal Processing, Vol. 67, No. 11, pp. 3013-3027, 2019.

[24] P. Ma, J. Li, X. Zhang, and G. Zhao, "Improved Unfolded Coprime Array Subject to Motion for DOA Estimation: Augmented Consecutive Synthetic Difference Co-array", IET Signal Process., Vol. 14, Issue 10, pp. 823-836, 2020.

[25] G. Qin and M. G. Amin, "Structured Sparse Array Design Exploiting Two Uniform 
Subarrays for DOA Estimation on Moving Platform", Signal Processing, Vol. 180, No. 3, 2021.

[26] C. L. Liu and P. P. Vaidyanathan, "Remarks on the spatial smoothing step in coarray MUSIC", IEEE Signal Process. Lett., Vol. 22, No. 9, pp. 1438-1442, 2015. 